subjects (CNTs). PBMCs, isolated from 9 among the SSc patients, were cultured on fibronectin-coated plates [5]. The non-adherent cells were removed and after 8 days (t8) of culture (standardized time), the adherent spindle shaped cells were lifted through incubation in $0.05 \%$ EDTA (ice-cold). Fibrocyte identification (at both t0, t8), was performed by FACS, using anti-CD45, anti-COL I, anti-CXCR4 and anti-HLA-DR monoclonal antibodies.

Results: FACS analysis revealed that, at basal time (t0), among the CD45+ cells, the percentage of fibrocytes, identified as triple positive (CD45+, COL $I_{+}$, CXCR4+) was $1.0 \pm 1.2 \%$ in SSc patients and $0.4 \pm 0.3 \%$ in healthy subjects (CNTs). In addition, the HLA-DR expression on fibrocytes in both SSc patients and CNTs showed low values $(22.1 \pm 21.1 \%$ and $7.1 \pm 6.1 \%$, respectively).

After 8 days (t8) of culture, fibrocytes presented adherent and spindle shaped morphology. Interestingly, the FACS analysis at t8 of culture, demonstrated that the percentage of SSc fibrocytes CD45+, COL I+, CXCR4+ increased up to $52.8 \pm 27.1 \%$, compared to basal time (t0), as well as strongly increasing the HLA-DR+ expression $(90.1 \pm 22.7 \%)$

Conclusions: Fibrocytes isolated from CPCs of SSc patients were confirmed to express CD45, COL I and CXCR4 molecules, but in very low percentage at the beginning. Already after 8 days of culture in proper conditions, the percentage of differentiated fibrocytes (CD45+, COL I+ and CXCR4+) from SSc patients, increased up to 50 times and the HLA-DR expression increased up to $68 \%$. Additional markers of progressive fibrocyte differentiation are now under test to further characterize the fibrocytes phenotype(s) of SSc patients vs healthy controls.

[1] Chesney J et al. Proc. Natl. Acad. Sci. 1997; 94:6307-6312.

[2] Bucala R. Mol Med 2015;21:S3-S5.

[3] Brunasso A.M. et al. F1000Research 2016:5:723

[4] Blakaj and Bucala. Fibrogenesis \& Tissue Repair 2012;5:S6

[5] Pilling D et al. J Immunol Methods 2009; 351:62-70.

Disclosure of Interest: None declared

DOI: 10.1136/annrheumdis-2017-eular.4963

\section{AB0184 CHROMOSOMAL ABERRATIONS ARE ASSOCIATED WITH DISEASE SEVERITY IN A MOUSE MODEL OF BLEOMYCIN-INDUCED SCLERODERMA}

R. Luisetto ${ }^{1}$, M. Beggio ${ }^{2}$, A. Scanu ${ }^{2}$, F. Leite de Oliveira ${ }^{3}$, E. Pigatto ${ }^{2}$, A. Peruffo ${ }^{4}$, E. Zanatta ${ }^{2}$, F. Oliviero ${ }^{2}$, P. Sfriso ${ }^{2}$, L. Punzi ${ }^{2}$, F. Cozzi ${ }^{2} .{ }^{1}$ Dept of Surgery, Oncology and Gastroenterology; ${ }^{2}$ Dept of Medicine- Rheumatology Unit, University of Padova, Padova, Italy; ${ }^{3}$ Institute of Biomedical Sciences, Federal University Rio de Janeiro, Rio de Janeiro, Brazil; ${ }^{4}$ Dept. of Comparative Biomedicine and Food Science, University of Padova, Padova, Italy

Background: Scleroderma (SSc) is a connective tissue disease characterized by immunomediated inflammation and fibrosis. The presence of autoantibodies against chromosome associate proteins,such as anti-centromere proteins and anti-topoisomerase I, was observed in SSc patients, however genotoxic events have never been evaluated (2).

Objectives: To assess the presence of aneuploidia, micronuclei (MN) and other chromosomal aberrations in peripheral lymphocytes in a bleomycin (BLM)-induced scleroderma mouse model.

Methods: BALB/c mice ( $\mathrm{n}=20$ per condition) were injected subcutaneously daily with BLM $2 \mathrm{mg} / \mathrm{kg}$ or PBS (control) for 14 days. Urine was collected weekly and proteinuria was measured. At day 15 mice were sacrificed and organs harvested for histological analysis. The MN assay was performed on cultured peripheral lymphocytes isolated from mice spleens. Cells were treated with cytochalasin B $(10 \mu \mathrm{g} / \mathrm{ml})$ for $24 \mathrm{~h}$ and a minimum of 1000 binucleated cells/mouse were scored following the criteria of Fenech (1). The antikinetochore (CREST) staining was used to determine the origin of $\mathrm{MN}$ observed.

Results: In BLM treated mice was observed huge perivascular lung fibrosis and significant skin involvement. 17 out of 20 mice developed acute renal involvement with mean proteinuria levels of $730 \pm 48 \mathrm{mg} / \mathrm{dl}$. In comparison with the control mice, a significant increase in MN number was observed in BLM treated mice $(57,8 \pm 4,4$ vs $6,3 \pm 0,6, p<0,05)$. CREST staining was higher in MN derived from BLM treated mice $(16,4 \pm 1,1$ vs $3,7 \pm 0,7, p<0,025)$, indicating that in this group lymphocyte $M N$ arised mainly from lagging chromosomes. In addition, an increased frequency of ring chromosome was observed in mice with greater skin fibrosis and renal involvement.A correlation between the presence of CREST stained MN and disease severity parameters as renal failure, lung and skin fibrosis was observed (respectively $R=0,4095 ; R=0,7507$ and $R=0,9471$ ).

Conclusions: This study provides experimental evidence that significant chromosomal aberrations seem to be related to the severity of disease in a mouse model of SSc. Further investigations on lymphocytes obtained from SSc patients could be matched with results observed in mouse model to confirm the relationship between severity of the disease and chromosomal abnormalities.

References:

[1] Fenech M. Cytokinesis-block micronucleus cytome assay. Nature Protocols 2006, 2(5): 1084-1199.

[2] Korman BD and Criswell LA. Recent advances in the genetics of systemic sclerosis: toward biological and clinical significance. Curr Rheumatol Rep 2015, 17(3): 21-34

Disclosure of Interest: None declared

DOI: 10.1136/annrheumdis-2017-eular.5043

\section{AB0185 ENDOTHELIN-1 STIMULATES THE PROFIBROTIC ALTERNATIVE ACTIVATED PHENOTYPE IN CULTURED HUMAN MACROPHAGES ISOLATED FROM SYSTEMIC SCLEROSIS} PATIENTS

S. Soldano ${ }^{1}$, P. Montagna ${ }^{1}$, R. Brizzolara ${ }^{1}$, A.C. Trombetta ${ }^{1}$, C. Corallo ${ }^{2}$, C. Pizzorni ${ }^{1}$, S. Paolino ${ }^{1}$, A. Sulli ${ }^{1}$, M. Ghio ${ }^{1}$, V. Smith ${ }^{3}$, N. Giordano ${ }^{2}$, M. Cutolo ${ }^{1}$. ${ }^{1}$ Research Laboratory and Academic Division of Clinical Rheumatology, Department of Internal Medicine, University of Genoa, Genoa;

${ }^{2}$ Medicine, Surgery and Neurosciences, University of Siena, Siena, Italy;

${ }^{3}$ Department of Rheumatology, Ghent University Hospital, Ghent University, Ghent, Belgium

Background: In the damaged tissue of systemic sclerosis (SSc) patients, the presence of immune inflammatory infiltrate, characterized by T-cells and macrophages, represents an important early pathological event in the fibrotic process (1)

In the macrophage population, the alternatively activated (M2) macrophages were observed in both peripheral blood and damage tissues of SSc patients and they participate in the fibrotic process exerting profibrotic effects, primarily by the production and release of transforming growth factor $\beta 1$ (TGF $\beta 1)(1,2)$. These cells are characterized by the expression of specific phenotype markers, such as mannose receptor (CD206) and scavenger receptors (CD204 and CD163), and the production of specific chemokines (i.e. macrophage derived chemokine, CCL-22) (3).

Endothelin-1 (ET1) plays an important role in the fibrotic process of SSc by inducing the profibrotic phenotype in endothelial cells and fibroblasts (4).

Objectives: To evaluate the ability of ET1 to upregulate the profibrotic gene expression profile characterizing the M2 macrophages in cultured monocytes/macrophages isolated from SSc patients.

Methods: Human monocytes were isolated from peripheral blood mononuclear cells of $4 \mathrm{SSc}$ patients (mean age 59 \pm 11 years, 2 females and 2 males, treated with vasodilator drugs) using a monocyte isolation kit. Cultured cells were maintained in RPMI growth medium for 24hrs and then treated with ET1 (100nM) for 6 days or treated for $1 \mathrm{hr}$ with $\mathrm{ET}$ receptor antagonist $\left(E T_{A B} R A\right.$, bosentan $\left.10 \mu \mathrm{M}\right)$ before stimulation with ET1. Cultured cells maintained in RPMI growth medium were used as untreated cells.

Gene and protein expression of M2 phenotype markers and TGF $\beta 1$ were evaluated by quantitative real-time polymerase chain reaction (qRT-PCR) and Western blotting (WB). Statistical analysis was carried out using a Mann-Whitney non-parametric test.

Results: In cultured SSc macrophages, ET1 induced the upregulation of the gene expression of CD206, CD204, CD163, and CCL22 compared to untreated cells. Moreover, ET1 increased the TGF $\beta 1$ gene expression compared to untreated cells. $\mathrm{ET}_{\mathrm{A} / \mathrm{B}} \mathrm{RA}$ partially contrasted the gene expression of CD204 and CD206. However, ET $A B A$ antagonized the ET1-mediated increase in the gene expression of CD163, CCL22 and TGF $\beta 1$. WB confirmed the results obtained by qRT-PCR.

Conclusions: Preliminary results showed the ability of ET1 to stimulate a profibrotic M2 phenotype in cultured human SSc macrophages characterized by the overexpression of TGF $\beta 1$, and this process is partially contrasted by the action of $E T_{A B B} R A$.

References:

[1] Higashi-Kuwata N et al. Arthrit Res Ther. 2010;12:R128.

[2] Stifano G et al. Curr Rheumatol Rep. 2016;18:2.

[3] Wynn TA et al. Nat Rev. 2013;496:445-55.

[4] Cipriani P et al. J Rheumatol. 2015;42:1808-16.

Disclosure of Interest: None declared

DOI: 10.1136/annrheumdis-2017-eular.5857

\section{AB0186 ASCA (ANTI-SACCHAROMYCES CEREVISAE ANTIBODY) IN SCLERODERMA}

A. Fedrigo, T.A.F.G. dos Santos, A. Bortoluzzi, T. Skare, R. Nisihara Rheumatology Unit, Hospital Universitário Evangelico de Curitiba, Curitiba, Brazil

Background: The majority of Scleroderma (SSc) patients have gastrointestinal involvement. Motility is usually compromised but few studies address permeability changes in the intestinal wall. ASCA is an antibody direct against mannan in the cell wall of Saccharomyces cerevisiae, the baker's yeast that is found mainly when the small bowel is involved in Chron's disease. Nowadays ASCA is regarded more as a marker of increased gastrointestinal permeability than as a serological autoimmune marker of intestinal inflammatory disease. ASCA levels (anti-Sacharomyces Cerevisae antibodies) are associated with increased intestinal permeability.

Objectives: At present we study the presence of ASCA in the serum of SSc patients aiming to know if there is increased intestinal permeability in this disease and if this is related to any clinical, demographic and treatment data.

Methods: Seventy four SSc patients and 57 healthy controls were studied for ASCA (IgG and IgA) positivity by ELISA. ASCA positivity was associated with demographic, clinical, severity index (Medsger score) and serological data in SSC patients.

Results: ASCA IgG levels were high in 26/74 (43.7\%) patients from SSc group and $1 / 57(5.2 \%)$ of controls $(p<0.001)$; IgA ASCA was high in $12 / 74(16.2 \%)$ from SSc group and $3 / 57(1.7 \%)$ of controls $(p=0.006)$. In univariated analysis IgG ASCA high levels associated positively with African background $(p<0.0001)$ and 